\title{
Computer modelling and simulation of the mechanical response of composite lattice structures
}

\author{
$\underline{\text { E.V. Morozov }}{ }^{\text {a }}$ and A.V. Lopatin ${ }^{\text {bc }}$ \\ ${ }^{a}$ School of Engineering and Information Technology, University of New South Wales at the Australian \\ Defence Force Academy, Canberra, Australia \\ ${ }^{b}$ Department of Aerospace Engineering, Siberian State University of Science and Technology, Krasnoyarsk, \\ Russia \\ ${ }^{c}$ Institute of Computational Technologies, Siberian Branch of Russian Academy of Sciences, Krasnoyarsk, \\ Russia \\ Email: e.morozov@adfa.edu.au
}

\begin{abstract}
Composite lattice shells are highly efficient and extensively used in various structural applications, such as rocket interstages, payload adapters for spacecraft launchers, fuselage components for aerial vehicles, and components of the deployable space antennas. The aim of this paper is to present an analytical approach based on the finite element discrete modelling that is capable of predicting mechanical behaviour of composite lattice structures with sufficient accuracy and, at the same time, is affordable in terms of computational expenses. This allows the modelling approach proposed in this work to enable the efficient solution of relevant design and design optimisation problems.
\end{abstract}

Structural analyses reported in the literature have been conventionally performed for composite cylindrical lattice shells. The paper investigates buckling behaviour of anisogrid composite lattice cylindrical, conical, and parabolic shells. The lattice shells are modelled as three-dimensional frame structures composed of curvilinear ribs subjected to the tension/compression, bending in two planes and torsion. The specialised finite-element model generation procedure (model generator/design modeller) is developed to control the orientation of the beam elements allowing the original twisted geometry of the curvilinear ribs to be closely approximated.

The generation of discrete models are presented and explained in detail for cylindrical and conical lattice shells. Buckling analyses are performed for the cylindrical and conical shells subjected to axial compressive loading, and for the structure composed of parabolic lattice shell loaded by transverse concentrated external load.

The effects of varying the length of the shells, the number of helical ribs and the angles of their orientation on the buckling behaviour of lattice structures are investigated. Critical buckling loads and corresponding buckling mode shapes are determined based on the modelling approach proposed in this work. The effects of parameters of the lattice structure on the values of critical buckling loads, mode shapes are examined using parametric analyses. Based on the computations, the angles of orientation of helical ribs delivering maximum critical loads for a number of particular structural designs are identified.

The results of these studies indicate that the modelling approach presented in this work can be successfully applied to the solution of design problems formulated for composite lattice shells.

Keywords: Composite lattice shells, finite-element modelling, spacecraft structures, buckling analysis, critical buckling loads 
Morozov and Lopatin, Computer modelling and simulation of the mechanical response of composite lattice structures

\section{INTRODUCTION}

Composite lattice cylindrical and conical shells made of unidirectional high-modulus carbon fibre reinforced plastics by continuous filament winding are increasingly used as structural components of spacecraft. Examples of such applications can be found in the designs of lattice anisogrid bodies of spacecraft and spokes of transformable/deployable umbrella like space antennas see e.g. Vasiliev et al. (2012). A typical design of a spacecraft with its body made in the form of a lattice cylindrical shell attached to a conical lattice payload adapter is shown in Figure 1. The lattice shells shown in the figure should provide the necessary level of mechanical structural performance during the launch and injection into orbit phases when the high intensity loadings are exerted on these structures. They normally include axial compression, transverse and pure bending, and torsion. Buckling under these loadings is one of the typical modes of failure of these shells.

The discrete models are normally built using finite-element modelling and could be composed of the beam, shell, or solid elements. Results of finite-element analyses of composite lattice shells can be found in the papers published by Fan et al. (2009), Frulloni et al. (2007), Hou and Gramoll (1998), Morozov et al. (2011a,b), and Zhang et al. (2009).

In this paper, an approach based on the finite element discrete modelling that is capable of simulating mechanical behaviour of composite lattice structures with sufficient accuracy and is affordable in terms of computational expenses is discussed.
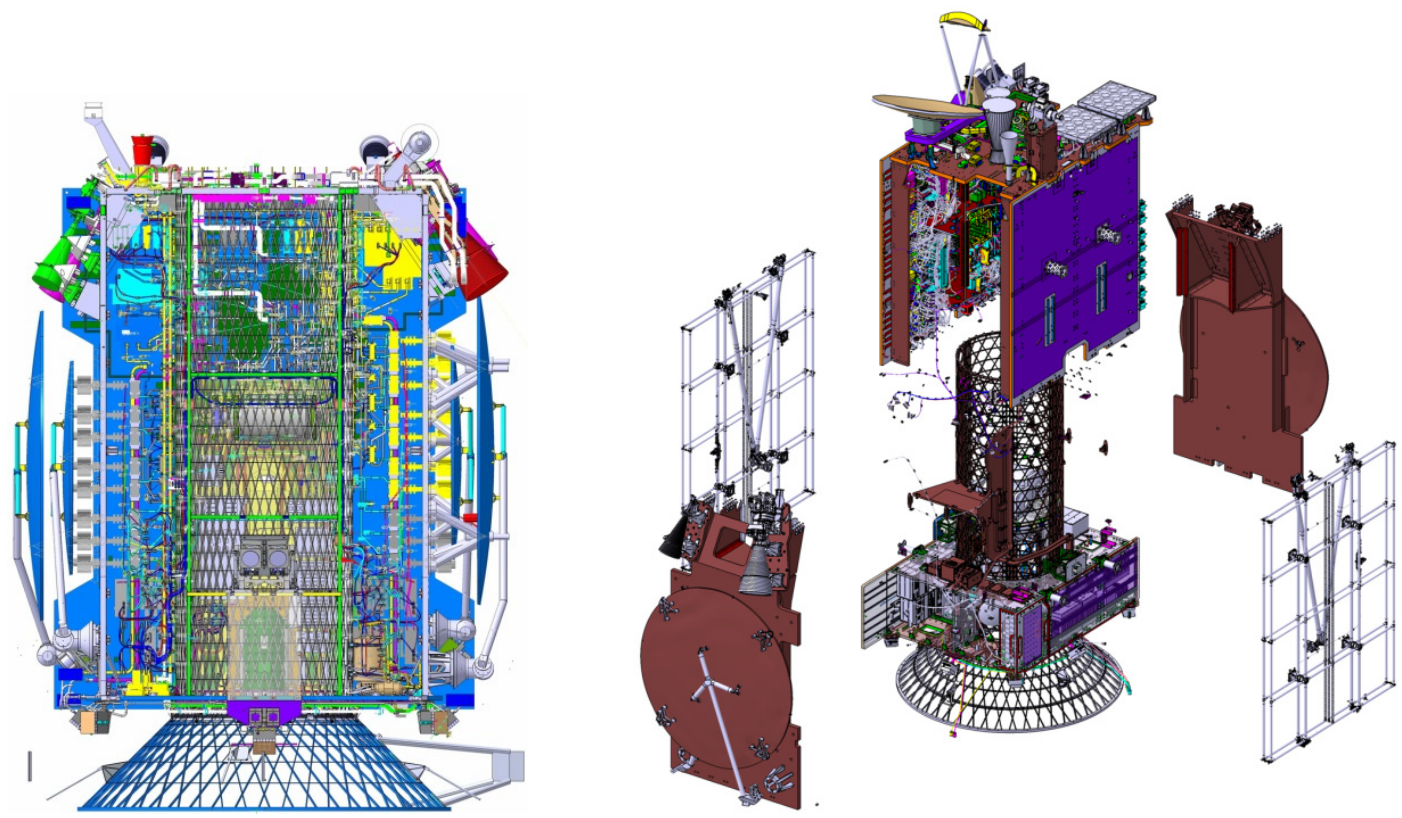

Figure 1. Spacecraft assembly including the composite lattice cylindrical body attached to the composite conical lattice adapter (Courtesy of ISS-Reshetnev Company).

\section{FINITE-ELEMENT MODELLING AND SIMULATION}

The lattice shells are modelled in this work as three-dimensional frame structures composed of the curvilinear ribs subjected to tension-compression, bending in two planes, and twisting. Using internal coding facilities of finite-element software packages, a model generator program can be developed to enable the geometry and finite-element model of the lattice shell to be automatically created. The generator/design modeller is capable of modelling the original geometry of the naturally twisted shapes of the helical ribs composing the lattice structure with maximum accuracy and authenticity. An additional advantage is that the size of the generated finite element model does not impede the efficiency of the computational process even for the lattice shells having a large number of ribs.

\subsection{Cylindrical lattice shells}

A composite lattice cylindrical shell is normally composed of two systems of filament wound ribs: a symmetric system of helical ribs and a system of circumferential ribs. The helical ribs make angles $\pm \phi$ with 
Morozov and Lopatin, Computer modelling and simulation of the mechanical response of composite lattice structures

the cylinder's generator line. The circumferential (hoop) ribs are passing through the middle points of the segments of helical ribs situated between the intersections as shown in Figure 2a. This arrangement of the ribs is referred to as the hexagonal lattice pattern. The geometry modelling of the lattice shell structure is based on four input variables, i.e. the overall length and diameter, $L$ and $D$, the helical angle, $\phi$, and the number of ribs, $n$, representing the system that has only one helical direction (either $+\phi$, or $-\phi$ ). The model is generated using a single typical unit cell shown in Figure 2a.

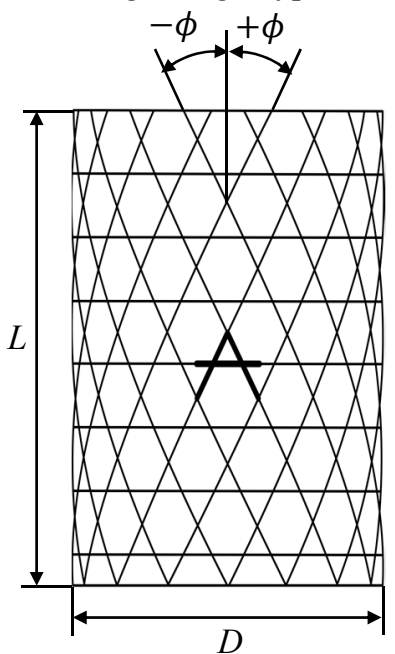

(a)

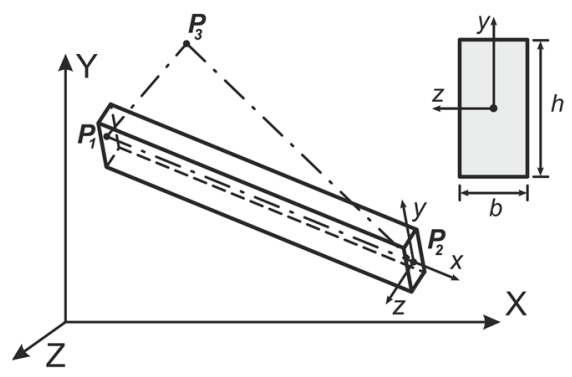

(b)

Figure 2. Lattice cylindrical shell (a) and linear finite beam element (b).

The lattice shell is modelled as a three-dimensional frame structure. The linear finite element having a $b \times h$ rectangular cross section and referred to the local coordinate frame $x y z$ (see Figure $2 \mathrm{~b}$ ) is employed to model the ribs. Each of the element nodes, $P_{1}$ and $P_{2}$ is characterised by six degrees of freedom, i.e. by three displacements and three rotations. The key point $P_{3}$, situated in the local plane $x y$, is used to orient the element with regard to the global frame $X Y Z$ as shown in Figure 2b. Using this element, the meshing of the typical unit cell is performed as shown in Figure 3. All the finite elements of the cell have the common reference key point $P_{3}$, which is located at the intersection of the axis of the cylinder and the plane of the circumferential rib. The use of this point as a reference allows the original natural twisted geometry of the curvilinear helical ribs to be closely approximated by the linear finite elements. Using rotation, copying, and translation of the meshed typical unit cell (see Figure 3), the complete finite element model of lattice cylindrical shell is generated. Then the ribs' characteristics are assigned including the sizes of the crosssections and the material properties, i.e. moduli of elasticity and densities.

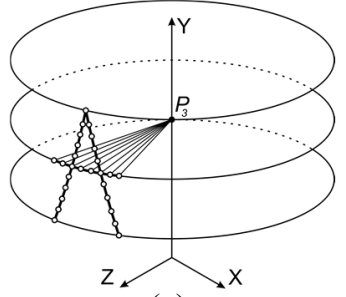

(a)

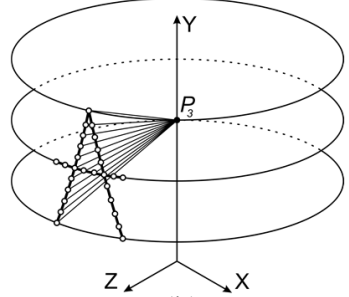

(b)

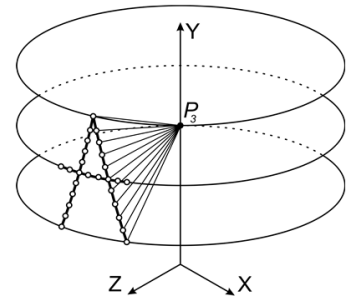

(c)

Figure 3. Meshing of the unit cell and positioning of the finite elements for the hoop ribs (a), and helical ribs with $+\phi(b)$ and $-\phi(c)$ angles of orientation.

The modelling algorithm has been realised in the form of the finite element model generator program and has been used for the automated finite element modelling of the lattice cylindrical shells.

Buckling analyses have been performed for the shells having a diameter $D=1 \mathrm{~m}$ and lengths $L=$ 0.5, 1.0 and $1.5 \mathrm{~m}$ using the FEA package COSMOS/M. The shells are composed of helical and hoop ribs of a $5 \times 5 \mathrm{~mm}$ square cross-section and made of unidirectional carbon-fibre reinforced plastic (CFRP) having the following properties: modulus of elasticity, $E_{x}=100 \mathrm{GPa}$, shear moduli, $G_{x y}=G_{x z}=5.5 \mathrm{GPa}, G_{y z}=$ $2.5 \mathrm{GPa}$, and density, $\rho=1500 \mathrm{~kg} / \mathrm{m}^{3}$. The analyses have been performed for a number of shells having the helical angles changing from $20^{\circ}$ to $50^{\circ}$ in the $5^{\circ}$ increments. The number of helical ribs of one helical 
Morozov and Lopatin, Computer modelling and simulation of the mechanical response of composite lattice structures

direction (either $+\phi$, or $-\phi$ ) $n=100$ has been kept the same for all the shells. The size of the beam finite elements was ranging from 10 to $20 \mathrm{~mm}$. For instance, the lattice structure of the shell with the length $L=$ $0.5 \mathrm{~m}$ and the angle $\phi=20^{\circ}$ has been modelled with $1040020-\mathrm{mm}$ long finite elements. The finite-element model of the lattice shell having $L=1.5 \mathrm{~m}$ and $\phi=50^{\circ}$ has been built of $8180020-\mathrm{mm}$ long finite elements. The bottom end of the shell is fully clamped and the axial load $\mathrm{P}$ is applied through the rigid ring attached to the top end. The critical loads $P_{c r}(L, \phi)$ obtained from the finite-element buckling analyses of the shells having various lengths and helical angles and subjected to axial compression are shown in Figure $4 \mathrm{a}$.

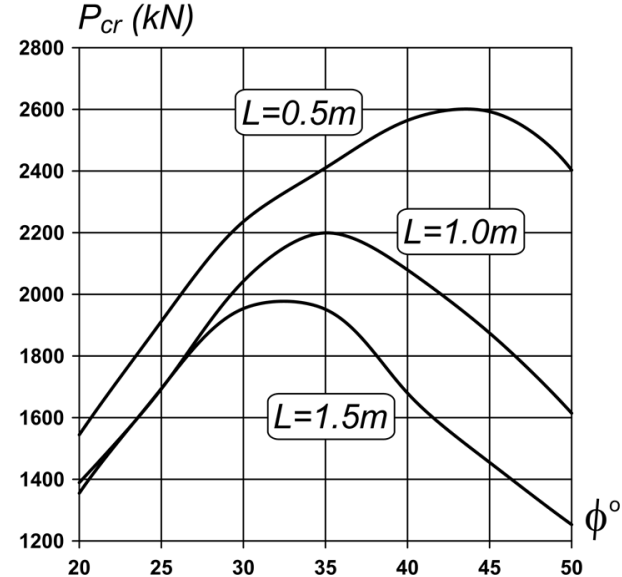

(a)

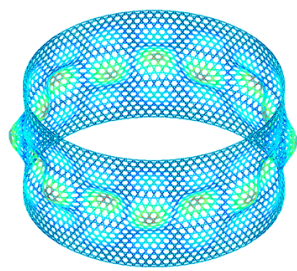

$L=0.5 \mathrm{~m}$

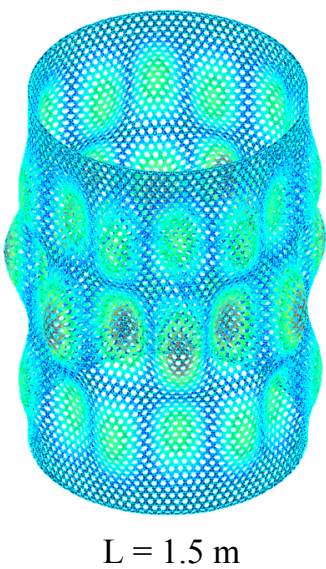

(b)

Figure 4. Function $P_{c r}(L, \phi)$ for the lattice shells under axial compression (a), buckling mode shapes for the shells with helical ribs wound at $\phi=30^{\circ}(\mathrm{b})$.

Examples of the buckling mode shapes for the shells with helical ribs wound at $\phi=30^{\circ}$ are shown in Figure 4b. Based on these computations, it was found that the shells with the helical ribs oriented at $\phi=30^{\circ}$ deliver the maximum weight efficiency. More computational results of the buckling analyses of cylindrical lattice shells subjected to transverse bending, pure bending, and torsion can be found in Morozov et al. (2011a).

\subsection{Conical lattice shells}

Similarly to the cylindrical lattice shells, truncated lattice conical shells are composed of two symmetric systems of filament wound helical and circumferential (hoop) ribs as shown in Figure 5.
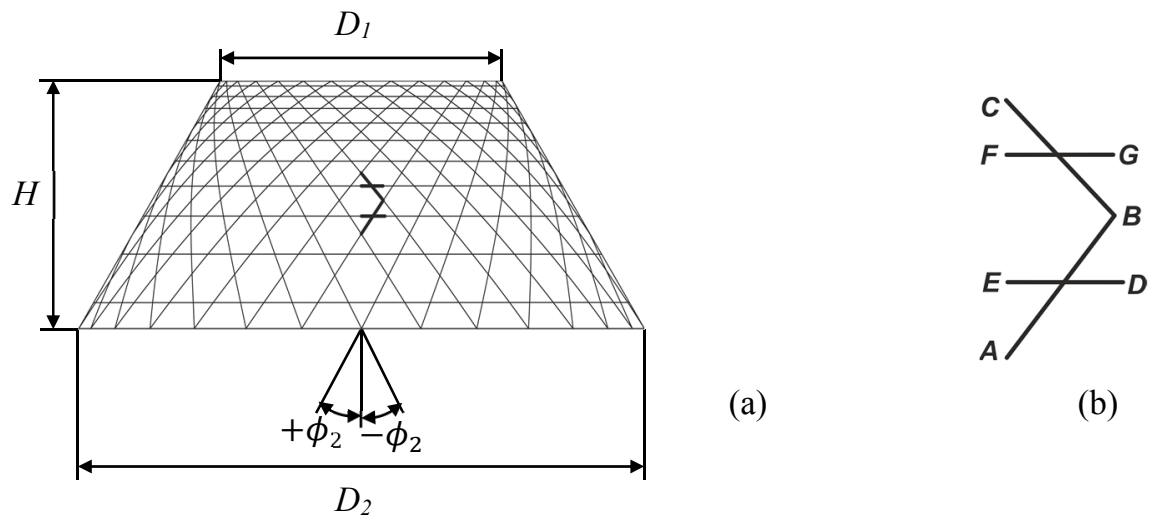

(a)

(b)

Figure 5. Conical lattice shell (a), and typical elementary unit (b).

The set of input data for the finite-element modelling includes: the height of the shell, $H$, diameters of the top and bottom sections, $D_{1}$ and $D_{2}$, number of the helical ribs of one direction (either $+\phi$, or $-\phi$ ), $n$ and the angle of the rib orientation at the bottom section of the shell $\phi_{2}$, (see Figure 5a). The finite-element model of the shell is generated using a typical elementary unit of the grid structure composed of two segments of the helical ribs $A B$ and $B C$ and two segments of the hoop ribs $E D$ and $F G$ as shown in Figure $5 \mathrm{~b}$. The lattice shell is modelled using the beam finite element referred to the local coordinate frame $x y z$ as shown in Figure $2 \mathrm{~b}$. Changing the location of the point $P_{3}$ within the frame $X Y Z$, the required orientation of the beam element can be achieved. 
Morozov and Lopatin, Computer modelling and simulation of the mechanical response of composite lattice structures

The typical elementary unit of the lattice structure (see Figure 5b) is meshed as shown in Figure 6a. All the beam elements of the unit $A B E D$ have the common reference key point $P_{3 E D}$ whereas all the elements of the unit BCFG have the common reference key point $P_{3 F G}$. Both points $P_{3 E D}$ and $P_{3 F G}$ are located at the intersections of the cone axis $Y$ with the planes of the hoop ribs $E D$ and $F G$, respectively (see Figure $6 a$ ). The selected positions of the key points allow the real geometry of the lattice shell composed of the twisted helical ribs to be reliably presented in the model.

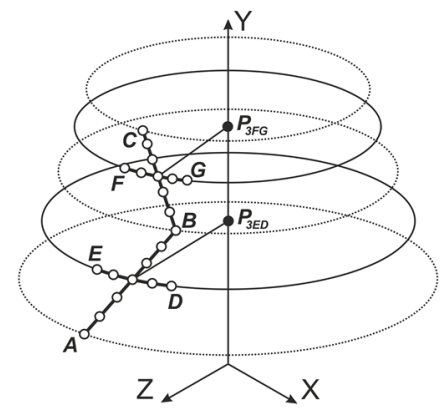

(a)
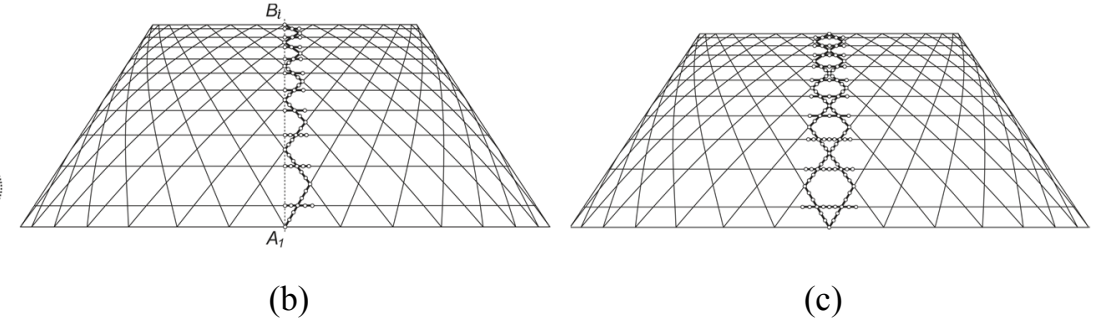

(c)

Figure 6. Meshing the elementary unit (a), placement of the units along the cone generator (b), basic finiteelement cell of the lattice structure.

The actual construction of the finite-element mesh is performed starting from the unit located at the bottom of the shell (see Figure 6b). The meshing is then carried on for the units located on top of each other along the cone generator line $A_{1} B_{i}$. As shown in Figure 6c, $i$ is the number of the last unit in this sequence located at the top of the cone. The helical ribs follow the geodesic trajectories which are defined by Clairaut's law: $r(Y) \cdot \sin \phi(Y)=$ const $=\left(D_{2} / 2\right) \sin \phi_{2}$, where $r(Y)$ is the current radius of the cone. The helical angle $\phi(Y)$ of the rib orientation changes according to this law from the bottom section to the top one. This change is taken into account when constructing the finite-element model from the elementary units as shown in Figure $6 \mathrm{~b}$. The meshed column group of these units shown in this figure is then mirror copied with respect to the cone generator line $A_{1} B_{i}$ creating the basic finite-element cell of the lattice structure shown in Figure 6c. Using copying and rotation of this cell around the cone axis, the complete finite-element model of the lattice conical shell is automatically generated. Once this procedure is complete, the meshed top and bottom end rings are added to the model. Input of the geometry parameters and material properties of the ribs completes the construction of the finite-element model. This algorithm has been implemented in the form of a finiteelement model generator program specifically developed for the problem under consideration. The generator was created using an internal FEA package code and has been applied to the automated construction of the finite-element models of conical lattice shells having various structural parameters (Morozov et al. (2011b)).

The buckling analyses have been performed for the filament wound composite conical lattice shells subjected to axial compression using COSMOS/M FEA package. The dimensions of the shells are: $H=0.6 \mathrm{~m}, D_{1}=$ $0.8 \mathrm{~m}, D_{2}=1.6 \mathrm{~m}$. The shells are made of the same unidirectional carbon-fibre reinforced plastic (CFRP) as the cylindrical shells considered earlier. The number of helical ribs of one helical direction (either $+\phi$, or $-\phi)$ is $n=60$. The analyses have been performed for the shells having the angles $\phi_{2}=$ $5^{\circ}, 10^{\circ}, 15^{\circ}, 20^{\circ}$ and $25^{\circ}$. Examples of finite-element models created for the angles $\phi_{2}=5^{\circ}, 15^{\circ}$, and $25^{\circ}$ are shown in Figure 7.

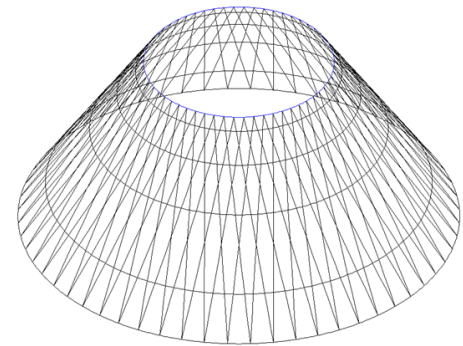

(a) $\phi_{2}=5^{\circ}$

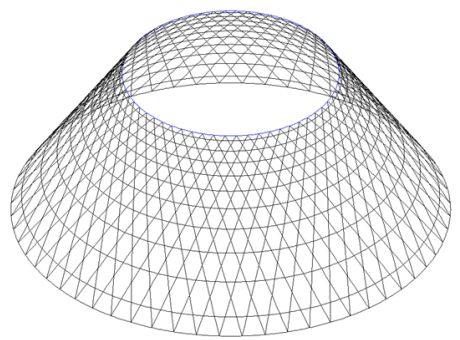

(b) $\phi_{2}=15^{\circ}$

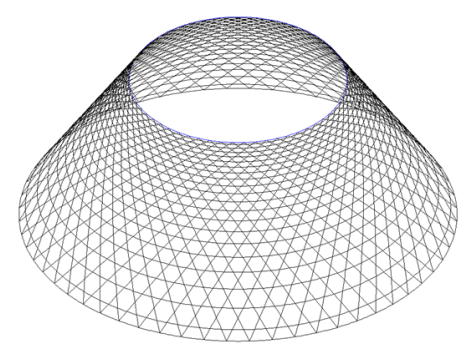

(c) $\phi_{2}=25^{\circ}$

Figure 7. Finite-element models of the lattice conical shells. 
Morozov and Lopatin, Computer modelling and simulation of the mechanical response of composite lattice structures

It is assumed, that all the ribs have the cross-sectional area of $16 \mathrm{~mm}^{2}$, whereas the height-to-width ratio $h / b$ (see Figure 2b) can vary. The following three combinations were considered in the analyses: (1) $h=2 \mathrm{~mm}$, $b=8 \mathrm{~mm}, h / b=0.25$; (2) $h=4 \mathrm{~mm}, b=4 \mathrm{~mm}, h / b=1$; (3) $h=8 \mathrm{~mm}, b=2 \mathrm{~mm}, h / b=4$. The lattice shell structures are modelled using the beam finite elements with the length ranging from 3.5 to 20 $\mathrm{mm}$ depending on the angle $\phi_{2}$. For instance, the lattice structure of the shell having the angle $\phi_{2}=10^{\circ}$ has been modelled with 1080010 to 20 -mm long finite elements. The finite-element model of the lattice shell having $\phi_{2}=25^{\circ}$ has been built of 252003.5 to $15-\mathrm{mm}$ long finite elements. The bottom end of the shell is fully clamped and the compressive force $P$ is applied through the rigid ring attached to the top section. The critical loads $P_{c r}\left(h / b, \phi_{2}\right)$ obtained from the simulations of the shells having various ratios $h / b$ and angles of the helical ribs' orientation at the bottom section, $\phi_{2}$ are shown in Table 1.

Table 1. Buckling loads $P_{c r}(\mathrm{kN})$ (axial compression).

\begin{tabular}{|l|c|c|c|}
\hline$\phi_{2}^{\circ}$ & $h=2 \mathrm{~mm} b=8 \mathrm{~mm}$ & $h=4 \mathrm{~mm} b=4 \mathrm{~mm}$ & $h=8 \mathrm{~mm} b=2 \mathrm{~mm}$ \\
\hline 5 & 13.863 & 34.416 & 44.334 \\
\hline 10 & 32.969 & 70.541 & 110.002 \\
\hline 15 & 60.601 & 109.475 & 179.714 \\
\hline 20 & 96.801 & 150.397 & 252.123 \\
\hline 25 & 139.542 & 192.213 & 316.574 \\
\hline
\end{tabular}

As expected, the shells with the highest ratio $h / b(h=8 \mathrm{~mm}$ and $b=2 \mathrm{~mm})$ are the most resistant to buckling. Typical buckling mode shapes are shown in Figure 8 . The shells with $h / b=0.25$ and 1 buckle in the mode characterised by formation of a few buckle waves at the bottom section which is typical for conventional homogeneous conical shells (see Figure 8a). For certain combinations of the helical angles and dimensions $h$ and $b$, shells buckle in the second mode characterised by the local buckling of ribs (see Figure $8 \mathrm{~b})$. This is typical for the lattice shells having $h / b=4$ and the angles $\phi_{2}=5^{\circ}, 10^{\circ}, 15^{\circ}$ and $20^{\circ}$.

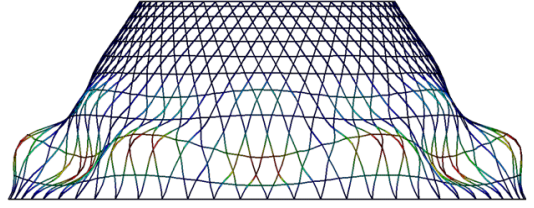

(a) Shell-like buckling

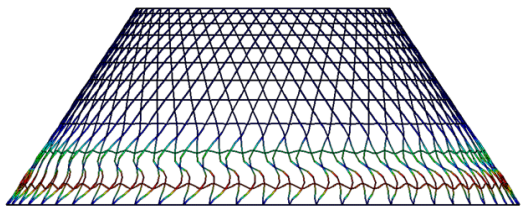

(b) Local buckling of ribs

Figure 8. Buckling mode shapes of conical lattice shells: (a) $\phi_{2}=15^{\circ}, h=b=4 \mathrm{~mm}$; (b) $\phi_{2}=15^{\circ}, h=$ $8 \mathrm{~mm}, b=2 \mathrm{~mm}$.

\subsection{Parabolic lattice shells}

The modelling approach presented in the previous sections was applied to the buckling analysis of a structure composed of parabolic composite lattice shells as shown in Figure 9.
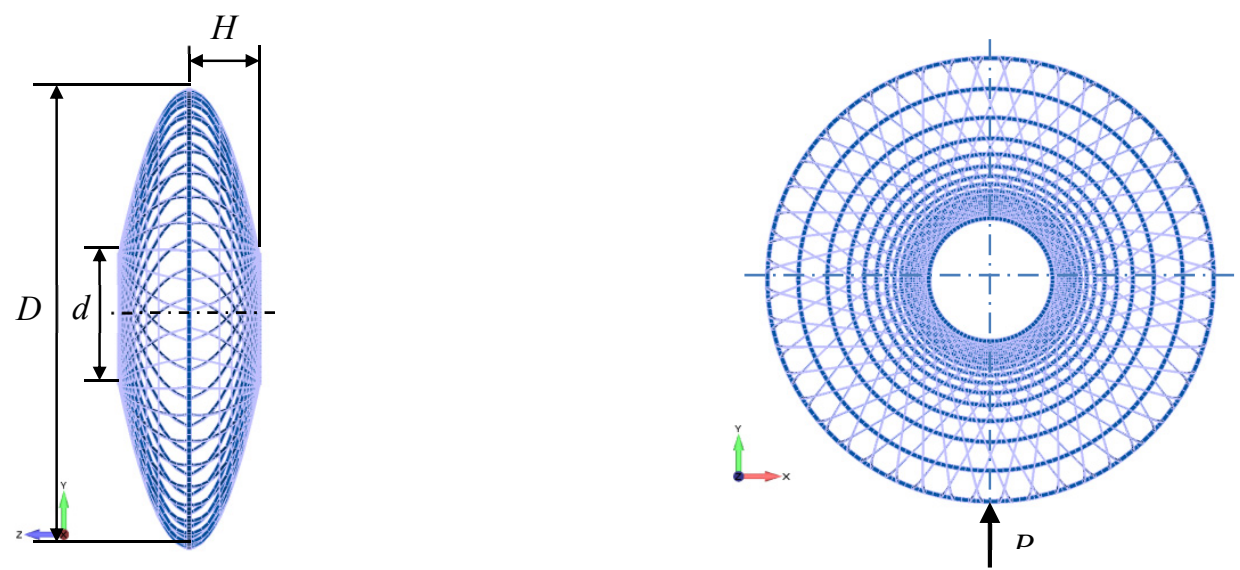

Figure 9. Parabolic composite lattice shell. 
Morozov and Lopatin, Computer modelling and simulation of the mechanical response of composite lattice structures

The shell is made of carbon fibre reinforced plastic by filament winding and loaded by concentrated load $P=$ $1000 \mathrm{~N}$ as shown in Figure 9. The shell dimensions are: $D=1 \mathrm{~m}, d=0.139 \mathrm{~m}, H=0.17 \mathrm{~m}$. The helical ribs have the following characteristics: $h=8 \mathrm{~mm}, \mathrm{~b}=2 \mathrm{~mm}, E=215.5 \mathrm{GPa}, G=3.5 \mathrm{GPa}, \rho=1650 \mathrm{~kg} / \mathrm{m}^{3}$. The hoop rings have $10 \times 2 \mathrm{~mm}$ rectangular cross sections and are made of the same material. The parabolic shells are joined by the internal and external rings with $10 \times 10 \mathrm{~mm}$ square cross sections. The structure is fully clamped at the inner diameter. The value of critical buckling load obtained from the finite element analysis is $P_{c r}=20.765 \mathrm{kN}$. The corresponding buckling mode shape is shown in Figure 10 .

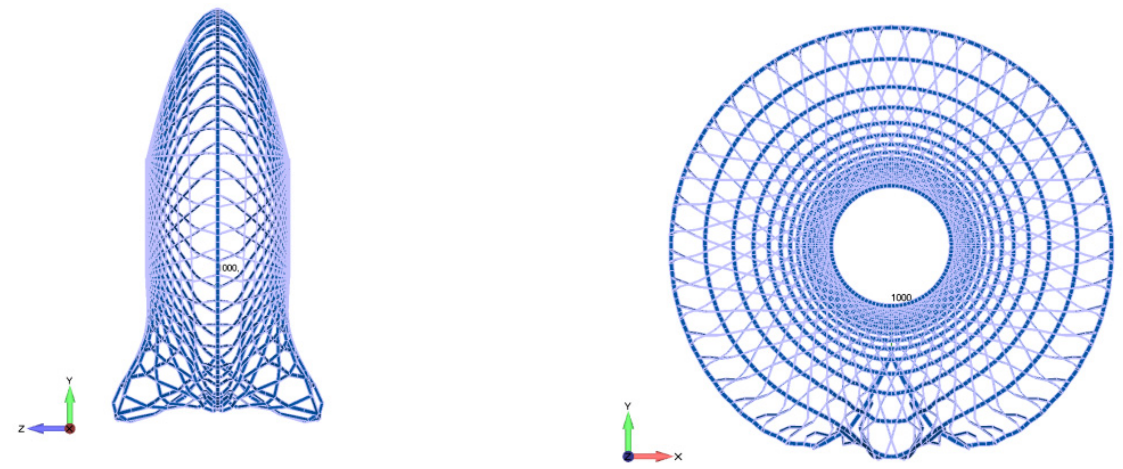

Figure 10. Buckling mode shape of the parabolic lattice shell.

\section{CONCLUSIONS}

A modelling approach based on the finite element discrete approximation is presented in this paper. The lattice shells are modelled as three-dimensional frame structures composed of curvilinear ribs subjected to the tension/compression, bending in two planes and torsion. The specialised finite-element model generation procedures developed in this work were applied to buckling analyses of composite cylindrical, conical, and parabolic shell structures. It is demonstrated that the simulations performed are capable of capturing major mechanical effects and predicting mechanical behaviour of composite lattice structures.

It was shown, based on the computational results that the modelling techniques considered in this paper provide effective tools for structural design and optimisation of composite structural components made in the form of filament wound lattice shells of various configurations. This is demonstrated for a number of particular structural applications.

\section{REFERENCES}

Fan H., Jin F., and Fang D. (2009). Uniaxial local buckling strength of periodic lattice composites. Materials and Design, 30, 4136-4145.

Frulloni E., Kenny J.M., Conti P., and Torre L. (2007). Experimental study and finite element analysis of the elastic instability of composite lattice structures for aeronautic applications. Composite Structures, 78, 519-528.

Hou A., and Gramoll K. (1998). Compressive strength of composite latticed structures. Journal of Reinforced Plastics and Composites, 17(5), 462-483.

Morozov E.V., Lopatin A.V., and Nesterov V.A. (2011a). Finite-element modelling and buckling analysis of anisogrid composite lattice cylindrical shells. Composite Structures, 93, 308-323.

Morozov E.V., Lopatin A.V., and Nesterov V.A. (2011b). Buckling analysis and design of anisogrid composite lattice conical shells. Composite Structures, 93, 3150-3162.

Vasiliev V.V., Barynin V.A., and Razin A.F. (2012). Anisogrid composite lattice structures - development and aerospace applications. Composite Structures, 94, 1117-1127.

Zhang Y., Xue Z., Chen L., and Fang D. (2009). Deformation and failure mechanisms of lattice cylindrical shells under axial loading. International Journal of Mechanical Sciences, 51, 213-221. 